\title{
Estimation of muscular forces from SSA smoothed sEMG signals calibrated by inverse dynamics-based physiological static optimization
}

\author{
F. Romero - F. J. Alonso - C. Gragera • \\ U. Lugrís • J. M. Font-Llagunes
}

Received: date / Accepted: date

\begin{abstract}
The estimation of muscular forces is useful in several areas such as Biomedical or Rehabilitation Engineering. As muscular forces cannot be measured in vivo non-invasively they must be estimated by using indirect measurements such as surface electromyography (sEMG) signals or by means of Inverse Dynamic (ID) analyses. This paper proposes an approach to estimate muscular forces based on both of them. The main idea is to tune a gain matrix so as to compute muscular forces from sEMG signals. To do so, a curve fitting process based on least-squares is carried out. The input is the sEMG signal filtered using Singular Spectrum Analysis technique. The output corresponds to the muscular force estimated by the ID analysis of the recorded task, a dumbbell weightlifting. Once the model parameters are tuned, it is possible to obtain an estimation of muscular forces based on sEMG signal. This procedure might be used to predict muscular forces in vivo outside the space limitations of the gait analysis laboratory.
\end{abstract}

Keywords Electromyography · Muscle Modelling · Muscular Forces · Inverse Dynamics Analysis · Singular Spectrum Analysis

F. Romero · F. J. Alonso · C. Gragera

Department of Mechanical, Energetic and Materials Engineering, University of Extremadura, Avda. de Elvas s/n, 06006, Badajoz, Spain

Tel.: +34-924-289600

Fax: +34-924-289601

E-mail: fromsan@unex.es

U. Lugrís

Department of Industrial Engineering II, University of La Coruña, Mendizábal s/n, 15403, Ferrol, Spain

J. M. Font-Llagunes

Dept. of Mechanical Engineering and Biomedical Engineering Research Centre (CREB), Universitat Politècnica de Catalunya, Avda. Diagonal 647, 08028, Barcelona, Spain 


\section{Introduction}

EMG is an experimental technique to detect and analyse the electrical signal that emanates from contracting muscles. Although the result cannot be directly considered as the real force exerted by the muscle, it can be useful to estimate it. Direct measurements of muscle forces are generally not feasible in a clinical setting and non-invasive methods, based on musculoskeletal modelling or indirect measurements, should be considered.

In the last years, efficient algorithms have been developed to estimate muscle forces. Inverse Dynamics (ID) can be used to estimate the net joint forces and moments applied to a joint, however, the contribution from muscles to generate these load is far more difficult to determine as there are more actuators than degrees of freedom, and different activation patterns may generate the same movement [1]. Therefore, optimization schemes are needed to solve such indeterminacy problem. Several optimization methods (static optimization, dynamic optimization, augmented static optimization) and optimization criteria (minimum metabolical cost of transport, minimum sum of muscle stresses, time-integral cost of activations, torque-tracking) are available in the literature [2-7]. The traditional ID computation method has been widely used to predict muscle forces but, unfortunately, the solution may not be physiologically consistent if static methods are used (where contraction dynamics is not considered), or may have a high computational cost for dynamic approaches. Besides, it is necessary a complete gait laboratory to collect the mentioned data, which implies increasing expenditures and facing space limitations.

Another approach to estimate muscle force is the direct prediction using the EMG signal. To do so, it is common to use EMG together with an appropriate muscle model [8-11]. In the last decades, the use of sophisticated computer algorithms and signal processing techniques has grown exponentially and now it is possible to decompose the EMG signal into the individual electrical activities of the muscle fibres in an efficient way $[12,13]$. Today, the decomposition approach promises to revolutionize clinical EMG and to provide a powerful tool for investigating the control schemes used by the nervous system to produce muscle contractions $[13,14]$.

The present work proposes a novel way to estimate muscular forces based on both approaches. Smoothed sEMG signals are calibrated by an inverse dynamicsbased physiological static optimization. Once the parameters of the estimation model were tuned, it was possible to compute muscular forces only from sEMG signals. As benchmarking maneuver, a dumbbell weightlifting task was carried out by a voluntary subject. The elbow flexo-extension movement is a simple task, fully documented in the literature [15-17], in which muscle parameters are well described too $[18,19]$.

\section{Methods}

The proposed approach comprises several steps (Fig. 1 (a)). Briefly, from the acquired data, two sets of raw data are obtained. On the one hand, kinematic data is processed and then used as input in the ID analysis to obtain the net elbow torque. Later, this net torque is distributed among muscles that actuate 
that joint by means of a physiological static optimization algorithm [20]. By the end of this step, an estimate of the muscular forces based on the acquired motion is available. On the other hand, sEMG, which is a low amplitude signal, must be amplified. As a consequence, noise will be amplified too. To distinguish between objective signal and noise in the raw sEMG data, a SSA smoothing algorithm is used. Once the signal is filtered, it is fitted to the ID-based muscular force curve using a least-squares approach. The obtained coefficients allow to estimate the muscular force using as input the filtered sEMG signal. The forces estimated using the calibration coefficients obtained through this procedure are compared and validated against an EMG-driven model (Fig. 1 (b)), as it has been widely done for muscle force assessment [8].

2.1 Motion capture analysis and elbow torque computation

As pointed out before, the first step is to obtain the net joint reaction forces and net joint torques at the elbow joint. To do so, an ID analysis is required. The human arm is modelled as a 3D multibody system composed of three rigid bodies linked by spherical joints. It has 9 degrees of freedom (DOF) and it is modelled using 39 dependent coordinates $(\mathrm{q}): 4$ points corresponding to the positions of all the spherical joints along with the center of mass of the hand plus two orthogonal unit vector for each rigid body. Both points and vectors are expressed using three Cartesian coordinates. Moreover, 3 sets of 3 angles that define the orientation of each segment with respect to the absolute frame are used, completing the 39 dependent coordinates set. Therefore a set of 39 constraint equations corresponding to rigid body restrictions and angular variable restrictions. A set of additional constraint equations is defined to complete the set of differential algebraic equation system. These additional 12 constraints correspond to the absolute angles between each rigid body and the ground (9 constraints) and the position of the shoulder joint (3 constraints). The dynamics of the $3 \mathrm{D}$ multibody system is then described by the equations of motion as:

$$
\left\{\begin{array}{c}
\mathbf{M} \ddot{\mathbf{q}}+\boldsymbol{\Phi}_{\mathbf{q}}^{T} \boldsymbol{\lambda}=\mathbf{Q} \\
\mathbf{\Phi}(\mathbf{q}, t)=\mathbf{0}
\end{array}\right.
$$

where $\mathbf{M}$ is the mass matrix of the system, $\ddot{\mathbf{q}}$ the accelerations vector, $\boldsymbol{\lambda}$ the Lagrange multipliers vector and $\mathbf{Q}$ the generalized forces vector. $\boldsymbol{\Phi}$ is the vector of constraint equations and $\mathbf{\Phi}_{\mathbf{q}}$ is the Jacobian matrix of the constraint equations. Using kinematic and anthropometric data in Eq. 1, the net joint reaction forces and net joint moments can be estimated. The kinematic data of the captured movement is obtained from the trajectories of a set of 7 markers attached to the human arm [21] (Fig. 2). This information is pre-processed to filter the high frequency noise and to correct the skin motion artifact, ensuring the kinematic consistency with the multibody model $[22,23]$. Once performed the ID analysis, the next step is to obtain muscular forces compatible with muscular physiology based on the obtained results. 
2.2 Muscle physiology. Hill-type muscle model

The dynamic behaviour of the muscle model can be expressed in terms of two cascaded differential equations, i.e., the excitation-to-activation dynamics (Eq. 2) and the activation-to-force dynamics (Eq. 3):

$$
\begin{gathered}
\dot{a}(t)=(u(t)-a(t)) \cdot\left(t_{1} u(t)+t_{2}\right) \\
\dot{F}^{m}(t)=g\left(a(t), F^{m}(t), l^{m}(t), i^{m}(t)\right)
\end{gathered}
$$

where $a(t)$ is the activation, $u(t)$ the excitation signal, $t_{1}$ and $t_{2}$ are time constants [24], and finally $F^{m}, l^{m}$ and $i^{m}$ are the muscular force, musculotendon length and lengthening velocity, respectively.

The muscle tissue's mechanical properties are described by the Hill-type muscle model $[25,26]$. It is composed by the contractile element $(C E)$, responsible of the active force generated in the muscle, and two non-linear passive springs: on the one hand, the series elastic element $(S E)$ that represents the elasticity of the actin-miosyn crossbridges [27] and, on the other hand, the parallel elastic element $(P E)$, that represents the passive elastic properties of the muscle fibres. The SE element can be neglected with little inaccuracy if the study does not involve shorttendon actuators $[3,26]$. In this work, the tendon is considered as a rigid element. According to this simplification, the force exerted by the $j$-th muscle $F_{j}^{m}(t)$ can be obtained directly using the expressions proposed by Thelen [28]. Table 1 lists the muscular parameters used in this work [18].

2.3 Estimation of muscle forces. Physiological static optimization (PSO) approach.

One of the most important problems in biomechanics is to distribute the calculated net joint moment into the different muscles acting on the same joint. As there are more unknowns than equations, an optimization scheme is needed to estimate muscular forces. It is well-known that static procedures solve the load sharing problem with lower computational cost than dynamic approaches, but muscle physiology is not considered. Halfway between them, physiological static optimization approaches are used to overcome the limitations of both methods. In this work, an approach proposed in a previous study is used [20]. This method comprises two steps. In the first one, the length and velocity of each musculotendon unit, $l^{m}$ and $i^{m}$ are obtained from generalized coordinates of the multibody model, as the origin and insertion points of the muscles are known. Then, the maximum force histories $F^{m, *}(t)$ compatible with contraction dynamics are calculated using the Eq. 3, supposing that muscle activations are maxima at every instant: $\mathbf{A}_{m}=\left[a_{1}, \ldots, a_{n}\right]^{T}=[1, \ldots, 1]^{T}$.

In the second step, muscle activations are calculated by solving an optimization problem where some physiological criteria are satisfied [3]. In this work, the sum of squared muscle stresses is minimized: 


$$
\begin{aligned}
& \operatorname{Min} J(\mathbf{A})=\sum_{j=1}^{n}\left(\frac{a_{j} F_{j}^{m, *}}{C_{j}}\right)^{2} \\
& \text { s.t. } \mathbf{R} \cdot\left(\mathbf{A} \mathbf{F}^{*}\right)=\mathbf{T} \\
& \quad 0 \leq a_{j} \leq 1
\end{aligned}
$$

where $F_{j}^{m, *} / C_{j}$ is the $j$-th muscle stress, and $C_{j}$ is the muscle physiological crosssectional area (PCSA). $\mathbf{A} \mathbf{F}_{m}^{*}=\left[a_{1} \cdot F_{1}^{m, *}, \ldots, a_{n} \cdot F_{n}^{m, *}\right]^{T}$ represents the muscular forces scaled by their activations, $\mathbf{R}$ is the vector of moment arms [29] and $\mathbf{T}$ is the net elbow torque. This non-linear optimization problem was solved using the sequential quadratic programming (SQP) function fmincon in MATLAB ${ }^{\circledR}$.

This optimization procedure comprises two constraints. The first one ensures that the sum of muscle moments must equal the net joint moment obtained previously. The second one is that activation must belong to the interval $[0,1]$, where $a_{j}=0$ represents no force exertion at muscle $j$, and $a_{j}=1$ represents full activation. The result of this optimization scheme is an estimation of the muscular forces based on the motion capture data.

\section{4 sEMG signal filtering using Singular Spectrum Analysis}

The acquired sEMG signal is rectified and, afterwards, filtered (Fig. 1 a)). Different methodologies have been established to that end, as Empirical Mode Decomposition [14], Wiener filtering [30] or Digital Butterworth filtering [31]. The approach presented in this work uses the Singular Spectrum Analysis (SSA) to filter sEMG signals. SSA is a non-parametric technique of time series analysis based on principles of multivariate statistics. This method decomposes a given time series into an additive set of independent time series. The resulting set of time series can be interpreted as trend representing the signal mean at each instant, a set of periodic series, and an aperiodic noise [32].

The SSA method builds a trajectory matrix (Hankel matrix) from the original time series in a process called embedding. This matrix consists of vectors obtained by means of a sliding window (of length $L$ ) that traverses the series. The trajectory matrix is then subjected to a Singular Value Decomposition (SVD). The result of the SVD is a sum of unit-rank matrices known as elementary matrices, each of which can be transformed into a reconstructed time series. Elementary matrices are no longer Hankel matrices, but an approximate time series may be recovered by taking the average of the diagonals (diagonal averaging). The resulting time series are called principal components [32]. The original time series is the sum of all these principal components. A more detailed description of the method can be found in [32].

To process the raw sEMG signal, the data was first rectified and then, the SSA algorithm was applied to obtain a smoothed signal. In this work, it was necessary to test different window lengths, the best results having been obtained for $L=400$. The singular spectrum (Fig. 3 (a)) indicates that the major contribution to the trend of the signal is given by the first component. The correlation matrix, in which each cell $\mathbf{C}_{i j}$ represents the correlation between principal components $i$ and $j$, gray-scale coded from black for 0 (no-correlation) to white for 1 (full correlation) shows (Fig. 3 (b)) that the first component is not correlated with the others, so 
that it may be interpreted as the smoothed EMG signal (Fig. 3 (c), top). The following components show different scale of grey, hence they might be interpreted as noise or crosstalk (Fig. 3 (c)). For further details of SSA applied to sEMG signal see $[33]$.

2.5 Muscle forces estimation based on calibrated sEMG signals

Once SSA-filtered sEMG signals have been obtained, it is possible to calculate a set of calibration coefficients that allow to estimate muscle forces from sEMG signals. To do so, a least-squares method is applied to sEMG signals.

Let $\mathbf{F}_{j}^{m}=F_{j, 1}^{m}, F_{j, 2}^{m}, \ldots, F_{j, n}^{m}$ be the predicted forces by ID analysis for the $j$-th muscle at $n$ time samples. In the same way, let $\mathbf{s}_{j}^{m}=s_{j, 1}^{m}, s_{j, 2}^{m}, \ldots, s_{j, n}^{m}$ be the processed sEMG signals for the $j$-th muscle. For each time sample $(1,2, \ldots, n)$, the force obtained by PSO can be related to the filtered sEMG signal as:

$$
\begin{aligned}
& F_{j, 1}^{m}=K_{j} \cdot h\left(s_{j, 1}^{m}\right)+C_{j} \\
& F_{j, 2}^{m}=K_{j} \cdot h\left(s_{j, 2}^{m}\right)+C_{j} \\
& \begin{array}{ll}
\vdots & \vdots \\
\vdots & \vdots
\end{array} \\
& F_{j, n}^{m}=K_{j} \cdot h\left(s_{j, n}^{m}\right)+C_{j}
\end{aligned}
$$

where $h\left(s_{j}^{m}\right)$ is a function of the processed sEMG signals that may not be linear on $s_{j}^{m}$. The resulting expression for $F_{j, i}^{m}$ must be linear on the calibration coefficients $K_{j}$ and $C_{j}$, the problem unknowns. Eq. 5 can be expressed in matrices terms as:

$$
\left(\begin{array}{c}
F_{j, 1}^{m} \\
F_{j, 2}^{m} \\
\vdots \\
F_{j, n}^{m}
\end{array}\right)=\left(\begin{array}{cc}
h\left(s_{j, 1}^{m}\right) & 1 \\
h\left(s_{j, 2}^{m}\right) & 1 \\
\vdots & \vdots \\
h\left(s_{j, n}^{m}\right) & 1
\end{array}\right)\left(\begin{array}{l}
K_{j} \\
C_{j}
\end{array}\right)
$$

or in a more compact manner as:

$$
\mathbf{F}_{j}^{m}=\mathbf{h}\left(\mathbf{s}_{j}^{m}\right) \cdot \mathbf{G}_{j}
$$

This system has more equations than variables. To obtain a square system of equations, each side of Eq. 7 is premultiplicated by $\left(\mathbf{h}\left(\mathbf{s}_{j}^{m}\right)\right)^{T}$ :

$$
\left(\mathbf{h}\left(\mathbf{s}_{j}^{m}\right)\right)^{T} \cdot \mathbf{F}_{j}^{m}=\left(\mathbf{h}\left(\mathbf{s}_{j}^{m}\right)\right)^{T} \cdot \mathbf{h}\left(\mathbf{s}_{j}^{m}\right) \cdot \mathbf{G}_{j}
$$

The solution of this system is unique and corresponds to the least-squares solution. This procedure is repeated for all the selected muscles. The obtained coefficient vector $\mathbf{G}_{j}$ contains the values to estimate muscular forces from sEMG signals at each time instant according to Eq. 7. Once those coefficients are tuned, it is possible to obtain an estimation of muscular forces based only on processed sEMG measurements.

Although, as proposed, the methodology is valid for any function $\mathbf{h}\left(\mathbf{s}^{m}\right)$ that satisfies the linearity on the coefficients, for simplicity the function $\mathbf{h}\left(\mathbf{s}^{m}\right)=\mathbf{s}^{m}$ has been used to scale and offset the sEMG signal with the calibration coefficients. Therefore, this function is used to phase sEMG data signal with the ID analysis muscle force. 


\subsection{Validation}

For an initial acquired movement (one flexo-extension cycle in 10 seconds without weight), calibration coefficients were calculated according to the proposed methodology. To assess the quality of the method, different movements $(1,2$ and 3 cycles in $10 \mathrm{~s}$ ) without load were recorded, and an estimation of the muscular forces was obtained using the previously calculated calibration coefficients. The cross-correlation was carried out by estimating the muscular forces for the same movements with a different dumbbell weight. The results were compared in each case with the ID-based force estimation (as the movement was also recorded) and an EMG-driven model [11]. This model uses an EMG-to-activation relationship $(A$-model $)$ to obtain the activations to be used in Eqs. 3. The EMG-to-activation relationship can be written as [11]:

$$
a_{j}(t)=\frac{e^{A u_{j}(t)}-1}{e^{A}-1}
$$

where $a_{j}(t)$ is the activation of the $j$-th muscle, $u_{j}(t)$ the processed sEMG signal of the $j$-th muscle, and $A$ a non-linear shape factor constrained to $-5<A<0$ (see [11] for upper limb muscles or [34] for lower limbs).

To evaluate the results, root mean squared error (RMSE) and normalized root mean squared error (NRMSE) were used as goodness-of-fit indicators:

$$
\begin{aligned}
R M S E & =\sqrt{\frac{1}{N} \sum_{j=1}^{N}\left(F_{A_{\text {model }}, j / C A L, j}-F_{I D}\right)^{2}} \\
N R M S E & =\frac{R M S E}{F_{A_{\text {model }}^{\text {max }} / C A L, j}^{\text {max }}-F_{A_{\text {model }}, j / C A L, j}^{\text {min }}}
\end{aligned}
$$

where $F_{A_{\text {model }}, j / C A L, j}$ corresponds to the A-model or the calibration-based force estimation for the $j$-th time instant, respectively, $F_{I D, j}$ is the estimated force through the ID-based force procedure, and $N$ is the number of time samples.

\section{Data acquisition}

The acquired movement consists of a dumbbell weightlifting with the right arm, from full extension at anatomical position to full flexion and return to the initial position. Different loads were used: no load for the calibration and correlation tests, and a dumbbells of $2.5 \mathrm{~kg}$, for the cross-correlation tests. The movement was performed at different velocities: 1,2 and 3 flexo-extension cycles in $10 \mathrm{~s}$. A total of 6 recordings were carried out for the experiment: one for calibration, five for validation. Seven reflective markers were placed according to Nigg and Herzog protocol [21]. The motion was recorded with 12 infra-red (IR) light cameras OptiTrack V100:R2 at $100 \mathrm{~Hz}$. An in-house developed software allows the synchronization of motion capture hardware with sEMG recording system. The software also allows to record kinematic data and to obtain the reconstructed trajectories. This software is based on the Camera Software Development Kit (SDK) provided by the manufacturer. The selected muscles for the experiment were the agonist 
muscles during flexion, namely biceps brachii and brachiorradialis. When the forearm is in a midposition between supination and pronation at the radioulnar joint, the brachioradialis acts as a strong elbow flexor, so this position for the forearm was selected for the task. The muscle activity was recorded on the superficial heads the biceps brachii and brachiorradialis. sEMG signals were recorded by means of standard passive surface electrodes with integrated ground (B\&L Engineering) following the recommended standard Surface EMG for a Non-Invasive Assessment of Muscles (SENIAM) [35]. The skin was abraded and cleaned with isopropyl alcohol. Then, a thin layer of conductive gel was extended along the point of application of the electrode, i.e., in the middle zone of the muscle, far from innervated an tendinosus zones [36] (see Fig. 2). sEMG signals were differentially amplified via commercial amplifier ( $B \& L$ Engineering) and acquired and exported to PC by means of NI-DAQ 6218-USB at a sampling frequency of $1 \mathrm{kHz}$. The processing of kinematic and sEMG signals was performed in MATLAB ${ }^{\circledR}$ using the multibody dynamics libraries developed at University of La Coruña, running on an Intel ${ }^{\circledR}$ Core $^{\text {TM }}$ i5 CPU at $3.20 \mathrm{GHz}$.

\section{Results and discussion}

The results obtained for the different combinations of load and movements are depicted in Fig. 4 and Table 2 for the biceps brachii, and in Fig. 5 and Table 3 for the brachiorradialis. Both muscles exert their action during flexion and extension, in this last case to smooth the movement. The curves related to triceps brachii are not drawn as sEMG did not show significant activity during the tests, as this muscle was not involved in the performed movement, i.e., the flexor muscles are the actuators during flexion but also during extension to stabilize the movement. In each cycle, two peaks are observed, and both correspond to the force developed at $L_{o p t}^{C E}$ : the first one when this value is reached during flexion and, the second one, during extension. The central minimum peak corresponds to the force exerted at full flexion. Goodness-of-fit indicators between estimated forces are listed in Tables 2 and 3. Regarding the results, it is observed that the curves obtained for brachiorradialis muscle are better than for biceps brachii, i.e., there is a better fit between curves for this last case than the first one. Regarding RMSE and NRMSE values, it is also observed, in general, minor divergences in the results listed for the brachiorradialis than the biceps brachii. The differences may be due to the electrode placement but also to the second peak observed in the contraction of the biceps brachii, which is related to the eccentric contraction of this muscle to control the extension movement. This peak in muscle activity may not be correctly reflected in the IDA, penalizing the calibration, and thus, the goodness-of-fit-indicators (almost $40 \%$ in most cases for biceps brachii). For the brachiorradialis muscle, this second peak is not observed and therefore the results show a better performance (around 20\% for the values for which is calibrated). The results could be improved by testing different window length or testing different reconstruction groups in the ssa method due to the great dependency of both methods with the de-noised EMG signal. The best results were obtained for the different movements with same weight with which the calibration coefficients were calculated, in this case under no load conditions. Nevertheless, as the dumbbell weight increases, correlation between the ID- and sEMG-based forces decreases. 
Thus, the proposed calibration scheme is only valid for the load conditions for which the calibration coefficients were calculated. Differences between ID- and sEMG-based forces may be due to the electromechanical delay [37-39] and to the simplification of the muscular model. Nevertheless, the proposed method could be useful in clinical applications. The calibration coefficients would be previously calculated in the laboratory for each muscle and subject, and then used to estimate muscular forces through sEMG measurements in any environment. ID-based methods as well as EMG-driven models must compute muscle-tendon lengths and contraction velocities to obtain an estimation of muscle forces, and, to do so, motion capture systems are required, which are not available in most environments. The independence of the motion analysis laboratory, once calibrated, is the main advantage of the proposed approach. However, the presented method has some limitations that should be considered. This method is valid for superficial muscles only, and many of them have more than one head. For deeper muscles, invasive procedures are required to get the EMG signals needed to properly quantify muscle activity or, at least, to estimate it from neighbouring superficial muscles [40]. Another drawback is the great dependency on the physiological parameters of the muscle, used in the ID analysis to estimate muscular forces, as some of them are difficult to obtain in-vivo. Thus, scale procedures and parameter optimization are necessary for each muscle. Finally, the use of SSA requires the selection of two parameters, namely the window length $L$ and the number of components to reconstruct the signal. The window length must be chosen in order to produce an adequate separability of the temporal series reconstructed from each elementary matrix. Regarding the components to reconstruct the signal, new algorithms based on SSA are needed to automate the filtering process [33]. Moreover, further experimental tests are required to extend the applicability of the method to different loads and different muscle groups.

\section{Conclusion}

This paper proposes a method to estimate muscular forces from SSA-smoothed sEMG signals calibrated by inverse dynamics-based physiological static optimization. The calibration coefficients allow to obtain an estimation of muscular forces from post-processed sEMG signals. The use of SSA filtering has been proven to be efficient to distinguish between the trend of the signal and the noise present and, therefore, it was selected as the filtering method. Traditional methods need data from motion capture system to estimate muscular forces and this may be unaffordable in terms of costs or mobility of the equipment. This method allows to estimate muscular forces from sEMG, once the parameters have been tuned for each subject. However, this method is restricted to the prediction of muscle forces of superficial muscles and is subject specific. Extensive testing on different subjects performing several physical activities is needed to adjust the muscular parameters and to validate the model. 


\section{Acknowledgements}

This work was supported by the Spanish Ministry of Economy and Competitiveness under project DPI2012-38331-C03, co-financed by the European Union through EFRD funds. The support is gratefully acknowledged.

\section{Conflict of interest}

All authors confirm that there are no competing interests associated with this publication.

\section{Ethical approval}

The experimental procedure was approved by the Ethics Committee of the University of La Coruña (CE-UDC), and confirmed by the Spanish Ministry of Economy and Competitiveness by the endorsement of the project DPI2012-38331-C03 that involves the three participant universities.

\section{References}

1. M. Ackermann. Dynamics and energetics of walking with prostheses. $\mathrm{PhD}$ thesis, University of Stuttgart, 2007.

2. A. Erdemir, S. McLean, W. Herzog, and A. J. van den Bogert. Model-based estimation of muscle forces exerted during movements. Clinical Biomechanics, 22(2):131-154, 2007.

3. M.T. Silva. Human motion analysis using multibody dynamics and optimization tools. PhD thesis, Instituto Superior Técnico (Universidade Técnica de Lisboa), 2003.

4. L.L. Menegaldo, A.T. Fleury, and H.I. Weber. A cheap optimal control approach to estimate muscles forces in musculoskeletal systems. Journal of Biomechanics, 39:1787-1795, 2006.

5. G Pipeleers, B Demeulenaere, I Jonkers, P Spaepen, Georges Van der Perre, A Spaepen, J Swevers, and Joris De Schutter. Dynamic simulation of human motion: numerically efficient inclusion of muscle physiology by convex optimization. Optimization and Engineering, 9(3):213-238, 2007.

6. J. A. C. Ambrosio and A. Kecskemethy. Multibody dynamics of biomechanical models for human motion via optimization. Multibody Dynamics, pages 245272, 2007.

7. Darryl G Thelen and Frank C Anderson. Using computed muscle control to generate forward dynamic simulations of human walking from experimental data. Journal of Biomechanics, 39(6):1107-1115, 2006.

8. D. G. Lloyd and T. F. Besier. An emg-driven musculoskeletal model to estimate muscle forces and knee joint moments in vivo. Journal of Biomechanics, 36(6):765-776, 2003.

9. Thomas S Buchanan, Michael J Moniz, Julius Dewald, and W Zev Rymer. Estimation of muscle forces about the wrist joint during isometric tasks using an EMG coefficient method. Journal of Biomechanics, 26(4):547-560, 1993. 
10. Stuart M McGill. A myoelectrically based dynamic three-dimensional model to predict loads on lumbar spine tissues during lateral bending. Journal of Biomechanics, 25(4):395-414, 1992.

11. Thomas S Buchanan, David G Lloyd, Kurt Manal, and Thor F Besier. Neuromusculoskeletal modeling: estimation of muscle forces and joint moments and movements from measurements of neural command. Journal of Applied Biomechanics, 20(4):367, 2004.

12. CJ De Luca, A Adam, R Wotiz, LD Gilmore, and SH Nawab. Decomposition of surface EMG signals. Journal of Neurophysiology, 96:1646-1657, 2006.

13. G. Wei, C. Wang, G. Tang, and F Tian. A wavelet-based method to predict muscle forces from surface electromyography signals in weightlifting. Journal of Bionic Engineering, 9:48-58, 2012.

14. Xu Zhang and Ping Xhou. Filtering of surface emg using ensemble empirical mode decomposition. Medical Engineering and Physics, 35:537-42, 2013.

15. AA Amis, D Dowson, and V Wright. Muscle strengths and musculoskeletal geometry of the upper limb. Engineering in Medicine, 8(1):41-48, 1979.

16. AA Amis, D Dowson, and V Wright. Analysis of elbow forces due to high-speed forearm movements. Journal of Biomechanics, 13(10):825-831, 1980.

17. Andris Freivalds. Biomechanics of the upper limbs: mechanics, modeling and musculoskeletal injuries. CRC press, 2011.

18. Katherine RS Holzbaur, Wendy M Murray, and Scott L Delp. A model of the upper extremity for simulating musculoskeletal surgery and analyzing neuromuscular control. Annals of Biomedical Engineering, 33(6):829-840, 2005.

19. Brian A Garner and Marcus G Pandy. Estimation of musculotendon properties in the human upper limb. Annals of Biomedical Engineering, 31(2):207-220, 2003.

20. Javier Alonso, Francisco Romero, Rosa Pàmies-Vilà, Urbano Lugrís, and Josep Maria Font-Llagunes. A simple approach to estimate muscle forces and orthosis actuation in powered assisted walking of spinal cord-injured subjects. Multibody System Dynamics, 28(1-2):109-124, 2012.

21. Benno Maurus Nigg and Walter Herzog. Biomechanics of the musculo-skeletal system, volume 192. Wiley New York, 1999.

22. MPT Silva and JAC Ambrósio. Kinematic data consistency in the inverse dynamic analysis of biomechanical systems. Multibody System Dynamics, 8(2):219-239, 2002.

23. FJ Alonso, JM Del Castillo, and P Pintado. Application of singular spectrum analysis to the smoothing of raw kinematic signals. Journal of Biomechanics, 38(5):1085-1092, 2005.

24. K.G.M. Gerritsen, A.J. van den Bogert, M. Hulliger, and R.F. Zernicke. Intrinsic muscle properties facilitate locomotor control: a computer simulation study. Motor Control, 2:206-220, 1998.

25. A V Hill. The heat of shortening and the dynamic constants of muscle. Proceedings of the Royal Society B Biological Sciences, 126(843):136-195, 1938.

26. F. Zajac. Muscle and tendon: properties, models, scaling and applications to biomechanics and motor control. Critical Reviews in Biomedical Engineering, 17:359-411, 1989.

27. Gary Tad Yamaguchi. Dynamic modeling of musculoskeletal motion: a vectorized approach for biomechanical analysis in three dimensions. Kluwer Academic Publishers Norwell, MA, 2001. 
28. Darryl G Thelen. Adjustment of muscle mechanics model parameters to simulate dynamic contractions in older adults. Journal of Biomechanical Engineering, 125(1):70-77, 2003.

29. John W Ramsay, Betsy V Hunter, and Roger V Gonzalez. Muscle moment arm and normalized moment contributions as reference data for musculoskeletal elbow and wrist joint models. Journal of Biomechanics, 42(4):463-473, 2009.

30. G. Aschero and P. Gizdulich. Denoising of surface EMG with a modified wiener filtering approach. Journal of Electromyography and Kinesiology, 20 (2):366-373, 2010.

31. R.G.T. Mello, L.F. Oliveira, and J. Nadal. Digital Butterworth filter for substracting noise from low magnitude surface electromyogram. Computer Methods and Programs in Biomedicine, 87(1):28-35, 2007.

32. Nina Golyandina, Vladimir Nekrutkin, and Anatoly A Zhigljavsky. Analysis of time series structure: SSA and related techniques, volume 90 . Chapman \& Hall, 2001.

33. F Romero, FJ Alonso, J Cubero, and G Galán-Marín. An automatic ssabased de-noising and smoothing technique for surface electromyography signals. Biomedical Signal Processing and Control, 18:317-324, 2015.

34. Massimo Sartori, Monica Reggiani, Cristiano Mezzato, and Enrico Pagello. A lower limb emg-driven biomechanical model for applications in rehabilitation robotics. In IEEE International Conference on Advanced Robotics, 2009. ICAR 2009., pages 1-7, 2009.

35. H. J. Hermens, B. Freriks, C. Disselhorst-Klug, and G. Rau. Development of recommendations for semg sensors and sensor placement procedures. Journal of Electromyography and Kinesiology, 10(5):361-374, 2000.

36. Eleanor Criswell. Cram's introduction to surface electromyography. Jones \& Bartlett Publishers, 2010.

37. PR Cavanagh and PV Komi. Electromechanical delay in human skeletal muscle under concentric and eccentric contractions. European Journal of Applied Physiology and Occupational Physiology, 42(3):159-163, 1979.

38. EJ Vos, MG Mullender, and GJ van Ingen Schenau. Electromechanical delay in the vastus lateralis muscle during dynamic isometric contractions. European Journal of Applied Physiology and Occupational Physiology, 60(6):467-471, 1990.

39. Shi Zhou, David L Lawson, William E Morrison, and Ian Fairweather. Electromechanical delay in isometric muscle contractions evoked by voluntary, reflex and electrical stimulation. European Journal of Applied Physiology and Occupational Physiology, 70(2):138-145, 1995.

40. Luciano L Menegaldo and Liliam F Oliveira. The influence of modeling hypothesis and experimental methodologies in the accuracy of muscle force estimation using EMG-driven models. Multibody System Dynamics, 28(1-2):21-36, 2012 . 


\begin{tabular}{ccccc}
\hline Muscle & $\begin{array}{c}\mathrm{C} \\
{\left[\mathrm{cm}^{2}\right]}\end{array}$ & $\begin{array}{c}F_{i s o}^{\max } \\
{[N]}\end{array}$ & $\begin{array}{c}l_{\mathrm{CE}}^{\text {opt }}[\mathrm{cm}] \\
{\left[{ }^{\circ}\right]}\end{array}$ & $\begin{array}{c}\alpha \\
{\left[{ }^{\circ}\right]}\end{array}$ \\
\hline $\mathrm{BB}$ & 4.5 & 624.3 & 11.6 & 0 \\
$\mathrm{BR}$ & 7.1 & 987.3 & 8.6 & 0 \\
$\mathrm{~TB}^{1}$ & 5.7 & 798.5 & 13.4 & 12 \\
$\mathrm{~TB}^{2}$ & 1.9 & 261.3 & 17.3 & 13.3 \\
\hline
\end{tabular}

Table 1 Muscle parameters [18]. BB: Biceps Brachii, BR: Brachiorradialis, TB ${ }^{1}$ : Triceps Brachii (Long Head), TB ${ }^{2}$ : Triceps Brachii (Lateral Head). C is the physiological crosssectional area, $F_{i s o}^{\max }$ is the maximum isometric force, $l_{C E}^{o p t}$ is the contractile element optimal length for maximum force production and $\alpha$ is the pennation angle. 


\begin{tabular}{|c|c|c|c|c|}
\hline \multicolumn{5}{|c|}{ Biceps Brachii } \\
\hline \multicolumn{5}{|c|}{1 cycle -10 seconds } \\
\hline & \multicolumn{2}{|c|}{$0 \mathrm{~kg}$} & \multicolumn{2}{|c|}{$2.5 \mathrm{~kg}$} \\
\hline & RMSE & NRMSE & RMSE & NRMSE \\
\hline$F_{I D}-F_{C A L}$ & 95.0777 & 0.3722 & 93.7603 & 0.3075 \\
\hline$F_{I D}-F_{A_{\text {model }}}$ & 99.1165 & 0.3881 & 97.4638 & 0.3197 \\
\hline \multicolumn{5}{|c|}{2 cycle -10 seconds } \\
\hline$F_{I D}-F_{C A L}$ & 82.4115 & 0.5663 & 144.5576 & 0.3841 \\
\hline$F_{I D}-F_{A_{\text {model }}}$ & 78.1776 & 0.5372 & 154.9058 & 0.4416 \\
\hline \multicolumn{5}{|c|}{3 cycle -10 seconds } \\
\hline$F_{I D}-F_{C A L}$ & 67.4955 & 0.3876 & 226.0388 & 0.3620 \\
\hline$F_{I D}-F_{A_{\text {model }}}$ & 67.4386 & 0.3873 & 227.6443 & 0.3646 \\
\hline
\end{tabular}

Table 2 Biceps Brachii results. Goodness-of-fit indicators: Root Mean Squared Error (RMSE) and Normalised Root Mean Squared Error (NRMSE) 


\begin{tabular}{ccccc}
\multicolumn{5}{c}{ Brachiorradialis } \\
\hline & \multicolumn{3}{c}{1 cycle -10 seconds } \\
& \multicolumn{3}{c}{$\mathrm{kg}$} & \multicolumn{2}{c}{$2.5 \mathrm{~kg}$} \\
\cline { 2 - 5 } & RMSE & NRMSE & RMSE & NRMSE \\
\hline$F_{I D}-F_{C A L}$ & 16.5069 & 0.1927 & 24.0990 & 0.1190 \\
\cline { 2 - 5 }$F_{I D}-F_{A_{\text {model }}}$ & 16.0002 & 0.1868 & 17.9639 & 0.0887 \\
\hline & 2 cycle -10 seconds & \\
\hline$F_{I D}-F_{C A L}$ & 25.9983 & 0.4792 & 53.1037 & 0.2364 \\
\cline { 2 - 5 }$F_{I D}-F_{A_{\text {model }}}$ & 21.2554 & 0.3918 & 22.4934 & 0.1002 \\
\hline & 3 cycle -10 seconds & \\
\hline$F_{I D}-F_{C A L}$ & 19.0029 & 0.3545 & 59.9189 & 0.1656 \\
\cline { 2 - 5 }$F_{I D}-F_{A_{\text {model }}}$ & 17.0461 & 0.3180 & 27.8765 & 0.0770 \\
\hline
\end{tabular}

Table 3 Brachiorradialis results. Goodness-of-fit indicators: Root Mean Squared Error (RMSE) and Normalised Root Mean Squared Error (NRMSE) 

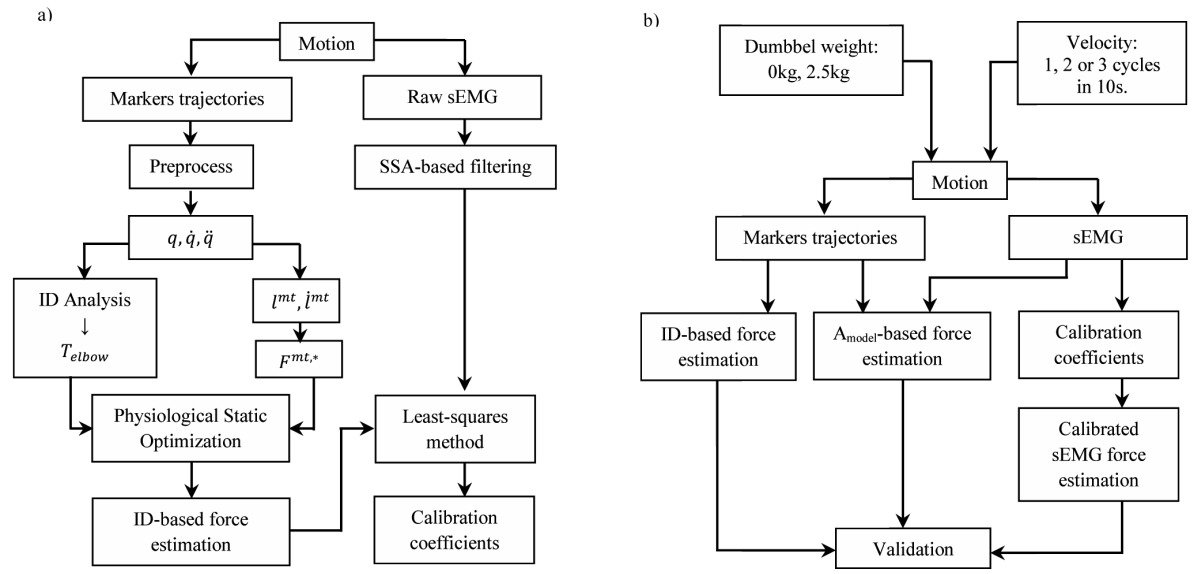

Fig. 1 a) Flowchart of the applied methodology to obtain the calibration parameters to estimate muscular forces from on sEMG signals. b) Validation procedure: selection of the dumbbell weight and movement velocity and comparison of the estimated muscle forces from ID analysis, A-model and calibration approaches. 


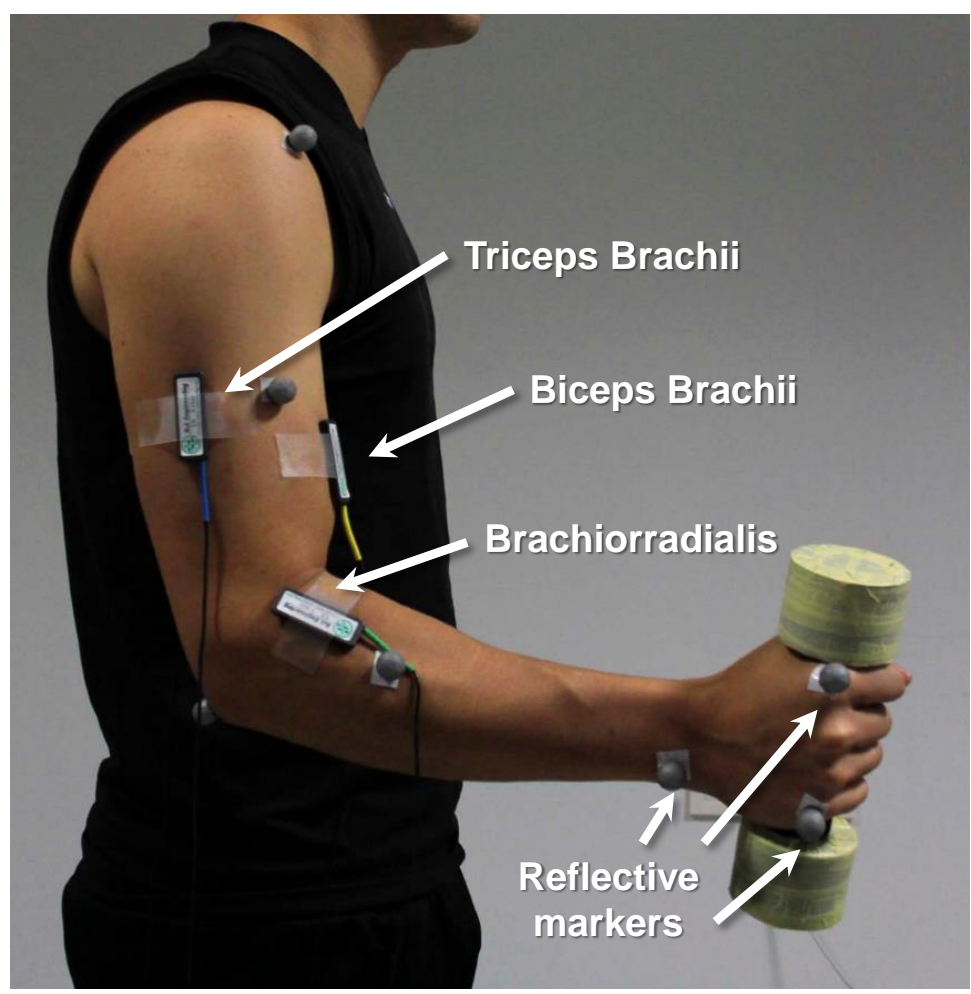

Fig. 2 Experimental setup with reflective markers and sEMG electrodes. 

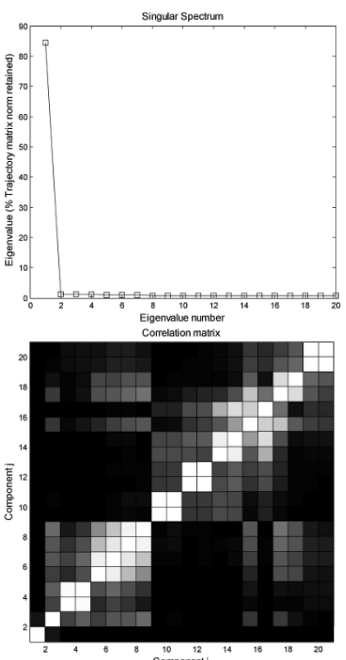

c)
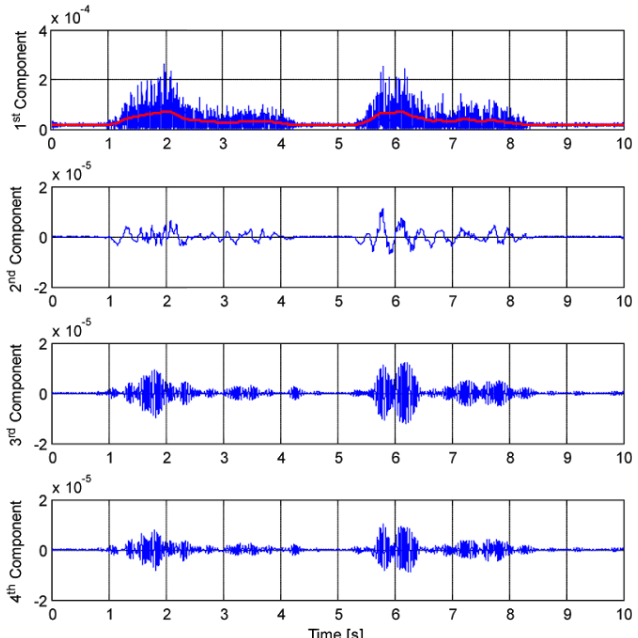

Fig. 3 a) Singular spectrum. For a better view, the figure is focused on the first 20 components. b) Correlation matrix focused on the first 20 components. c) Rectified raw signal and filtered signal corresponding to the first component (red line, top graphic) and the three following components. 

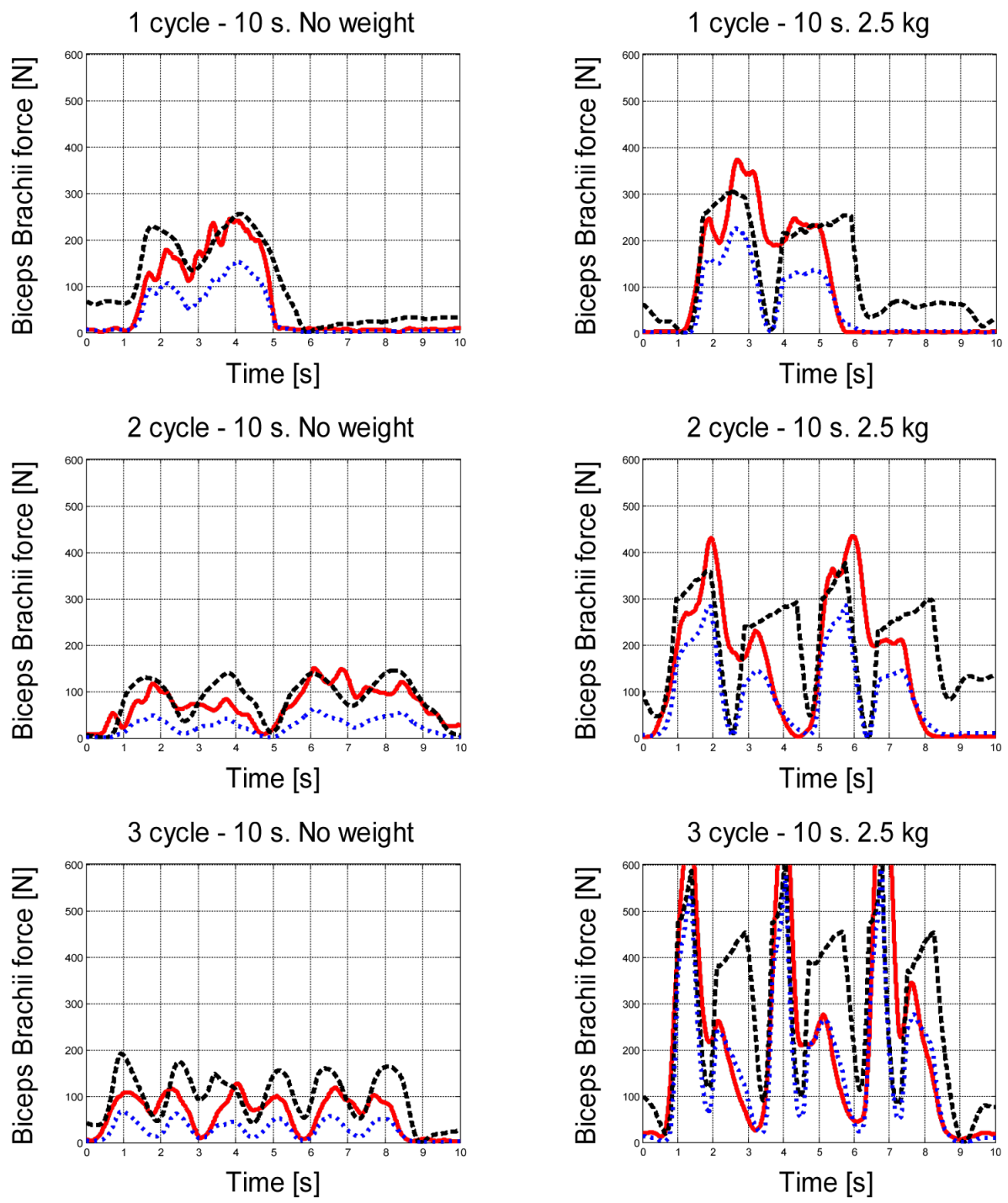

Fig. 4 Biceps Brachii results. Estimation of forces through the different methods. Black dashed line: ID-based forces. Red solid line: sEMG calibrated forces. Blue dotted line: A-model based forces. 

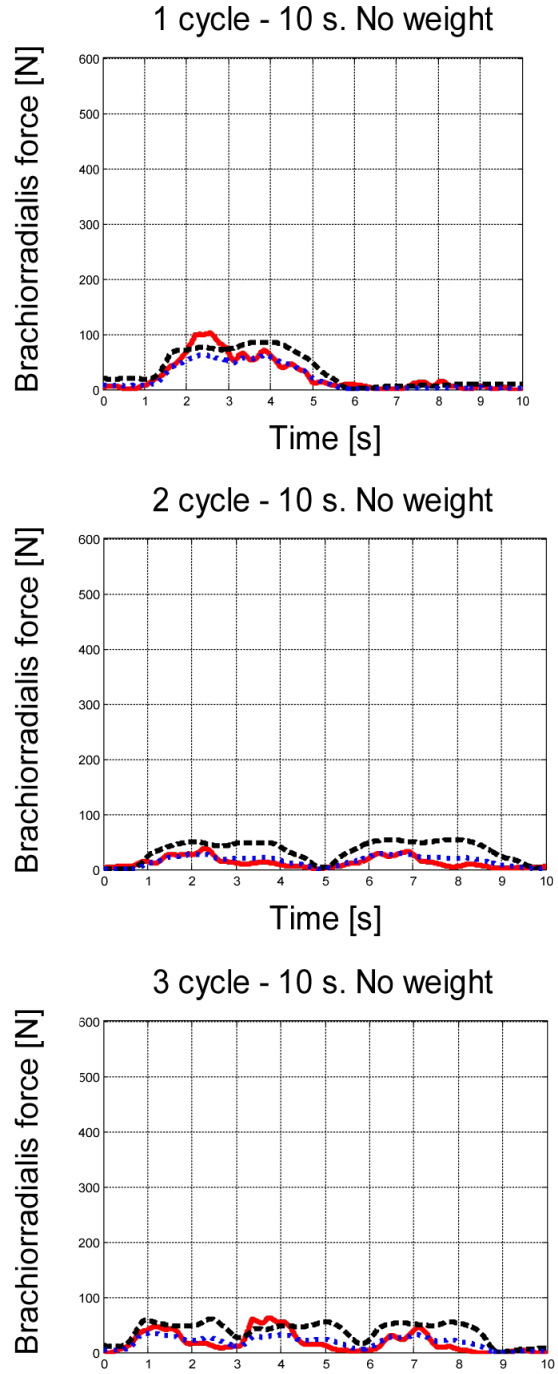

Time [s]

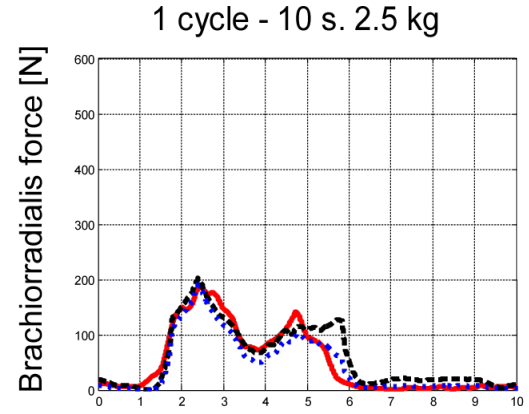

Time [s]
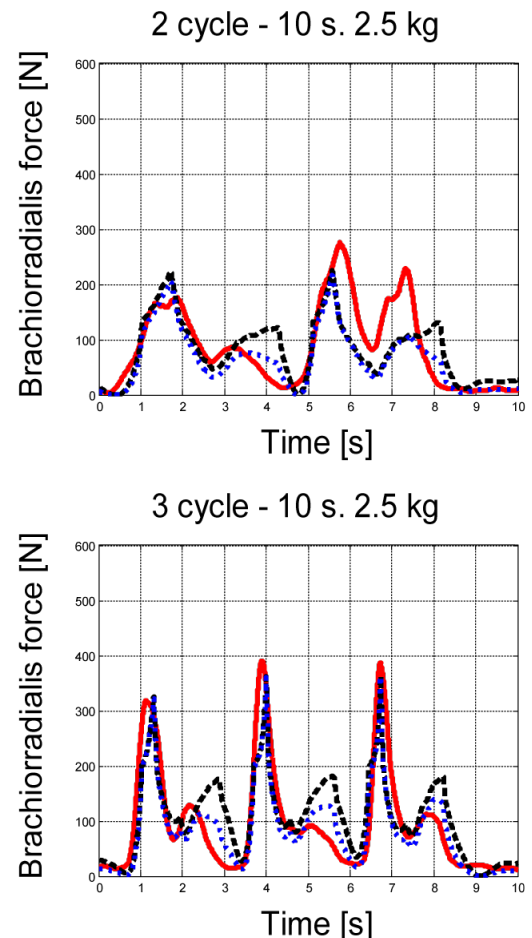

Time $[\mathbf{s}]$

Fig. 5 Brachiorradialis results. Estimation of forces through the different methods. Black dashed line: ID-based forces. Red solid line: sEMG calibrated forces. Blue dotted line: A-model based forces. 